\title{
Lateral tarsal strip versus Bick's procedure in correction of eyelid malposition
}

\author{
Kaveh Vahdani ${ }^{1} \cdot$ Rebecca Ford $^{1} \cdot$ Helen Garrott $^{1} \cdot$ Vladimir Theodor Thaller $^{2}$ \\ Received: 20 November 2017 / Revised: 23 December 2017 / Accepted: 3 January 2018 / Published online: 21 February 2018 \\ (c) The Royal College of Ophthalmologists 2018
}

\begin{abstract}
Objectives To compare the functional and anatomical outcomes of lateral tarsal strip (LTS) with Bick's procedure in treatment of eyelid malposition.

Methods A retrospective, consecutive case series of patients who underwent LTS and Bick's procedure for all types of involutional lower eyelid malposition, at two centers between January 2012 and 2015. Statistical analyses of differences between groups were performed using the Fisher's exact test to compare non-continuous variables and Mann-Whitney $U$ test for continuous variables.

Results A total of 641 procedures (557 LTS and 84 Bick's) were performed on 504 patients (137 bilateral) by 7 consultants and their trainees. The study cohort included 286 males and 218 females. The mean age was 76.2 years (median 78 ). The mean follow-up was 13.07 months (median 7, range 0.5-58 months). The indications for surgery included ectropion (43.2\%), entropion (39.9\%), eyelid laxity (12.3\%), floppy eyelid syndrome (2.7\%), and others (1.9\%). At last follow-up, the LTS group achieved $89.1 \%$ anatomical success (total + partial correction) compared to $100 \%$ in Bick's group $(P<0.001)$. Functional improvement was $82 \%$ and $95 \%$ in LTS and Bick's groups, respectively $(P=0.002)$. Consultants and trainee doctors achieved comparable outcomes within each group. Complications were relatively minor with no major long-term sequelae, nor any statistically significant difference between the two groups in terms of frequency of adverse events (16.9\% vs $14.2 \%, P=0.929)$. The reoperation rate was $9 \%$ in the LTS group during the study period, compared to none in the Bick's group $(P=0.001)$.

Conclusions This study compares the outcomes of the largest reported cohort of eyelid malposition surgery analyzing the two different techniques for lid margin shortening. Bick's procedure achieved statistically significant better anatomical and functional outcomes compared to LTS although the samples were unequal and not randomized.
\end{abstract}

\section{Introduction}

Eyelid malposition is a common problem. This encompasses a variety of conditions including ectropion, entropion, or generalized lid laxity. Horizontal eyelid margin laxity is an important factor that influences the eyelid position and orientation. Many surgical procedures have been described for addressing horizontal eyelid laxity including lateral tarsal strip (LTS), wedge excision, or

Kaveh Vahdani

kaveh.vahdani@nhs.net

1 Bristol Eye Hospital, Lower Maudlin Street, Bristol BS 1 2LX, UK

2 Royal Eye Infirmary, Derriford Hospital, Plymouth PL6 8DH, UK lateral Bick's shortening [1-4]. Lateral tarsal strip (LTS) is a widely used and successful procedure for management of horizontal laxity $[2,3]$. Bick's procedure (full thickness lid margin resection at the lateral canthus), which is a less wellknown technique, has the advantages of the LTS, as well as being a technically simpler operation [4]. The authors present the results of a large retrospective study, evaluating the effectiveness of these two techniques used for correction of the horizontal eyelid laxity.

\section{Methods}

This is a retrospective, comparative, consecutive case series of patients who underwent LTS and Bick's procedure for involutional ectropion, entropion, and other lower eyelid malposition, at two centers (Bristol Eye Hospital and Royal 
Eye Infirmary Plymouth) between January 2012 and 2015. The study was conducted in accordance to our institutional ethical standards. Data were collected from patient charts and electronic records.

Primary outcome measures were functional and anatomical success. Anatomical success was defined as complete (full correction) or partial (undercorrection or overcorrection) restoration of the normal eyelid/punctal position. Functional success was defined as improvement of the chief symptomatic complaint (complete resolution or partial resolution). Early and late post-operative complications, as well as reoperation rate was recorded during the follow-up period. Data were also collected on patient demographics, adnexal comorbidities, previous eyelid or lacrimal surgery, simultaneous procedures, and grade of the surgeon. The surgeries were performed by seven consultant oculoplastic surgeons and their trainees.

The standard repair technique for correction of the simple involutional ectropion consisted of LTS or modified Bick's \pm addition of medial spindle (MS) procedure in cases with marked medial ectropion [5], while simple involutional entropion was addressed with LTS or modified Bick's \pm everting sutures or lower lid retractor plication [6, 7]. Additional adjunctive procedures were performed, where applicable (Table 1). Below we describe our standard surgical technique for LTS and Bick's.

\section{Surgical technique for lateral tarsal strip}

- Horizontal skin and orbicularis incision, measuring approximately $5-10 \mathrm{~mm}$ at the lateral canthus (Fig. 1a), exposing the periosteum of the lateral orbital wall.

- Canthotomy (Fig. 1b) and cantholysis of the lower limb of the lateral canthal tendon (LCT) (Fig. 1c).

- Fashioning of the tarsal strip by gray line split with separation of the anterior and posterior lamellae of the lateral eyelid.

- Excision of the anterior lamella overlying the tarsal strip.

- Cautery and debridement of the tarsal conjunctival epithelium behind the tarsal strip.

- Excision of the muco-cutaneous junction at the lid margin.

- Lateral tarsal strip shortened to the desired length.

- Placement of one double-armed 5/0 polyglactin 910 (Coated VICRYL ${ }^{\circledR}$, Ethicon) suture on $1 / 4$ circle needle through the LTS and fixation to the periosteum of the lateral orbital rim (Fig. 1d).

- Lid angle reformation suture with $6 / 0$ polyglactin 910 buried suture into gray line.

- Skin and orbicularis closure with $6 / 0$ or $7 / 0$ polyglactin 910 sutures.
Table 1 Baseline characteristics

\begin{tabular}{|c|c|c|c|}
\hline Characteristics & $\begin{array}{l}\text { LTS } \\
(n=557)\end{array}$ & $\begin{array}{l}\text { Bick's } \\
(n=84)\end{array}$ & $P$ value \\
\hline Age (years) mean $\pm \mathrm{SD}$ & $76.1 \pm 11$ & $76.6 \pm 9.7$ & 0.94 \\
\hline Gender (F/M) & $175: 250$ & $43 / 36$ & 0.35 \\
\hline Diagnosis $(n(\%))$ & & & 0.43 \\
\hline \multicolumn{4}{|l|}{ Ectropion } \\
\hline Involutional & $224(40.2)$ & $30(35.7)$ & \\
\hline Recurrent & $17(3)$ & $2(2.4)$ & \\
\hline Other & $4(0.7)$ & - & \\
\hline All & $245(43.9)$ & $32(38.1)$ & \\
\hline \multicolumn{4}{|l|}{ Entropion } \\
\hline Involutional & $195(35)$ & $45(53.6)$ & \\
\hline Recurrent & $13(2.3)$ & $2(2.4)$ & \\
\hline Other & $1(0.2)$ & - & \\
\hline All & $209(37.5)$ & $47(56)$ & \\
\hline Lax eyelid syndrome & $76(13.6)$ & $3(3.6)$ & \\
\hline Floppy eyelid syndrome & $16(2.9)$ & $1(1.2)$ & \\
\hline Other & $11(2)$ & $1(1.2)$ & \\
\hline \multicolumn{4}{|c|}{ Simultaneous adjunctive procedures $(n(\%))$} \\
\hline Everting sutures & $117(21)$ & $1(1.2)$ & \\
\hline $\begin{array}{l}\text { Retractor plication - } \\
\text { transcutaneous }\end{array}$ & $82(14.7)$ & 45 (53.6) & \\
\hline $\begin{array}{l}\text { Retractor plication - } \\
\text { transconjunctival }\end{array}$ & 115 (20.6) & $9(10.7)$ & \\
\hline $\begin{array}{l}\text { Medial spindle type } \\
\text { procedure }\end{array}$ & 98 (17.6) & $5(5.9)$ & \\
\hline Punctoplasty & $91(16.3)$ & $1(1.2)$ & \\
\hline $\begin{array}{l}\text { Sub-orbicularis oculi fat } \\
\text { (SOOF) lift }\end{array}$ & $9(1.6)$ & - & \\
\hline Other & $50(8.9)$ & $7(8.3)$ & \\
\hline
\end{tabular}

\section{Surgical technique for modified lateral Bick's procedure}

- Full thickness 5-10 mm incision of the eyelid/lateral canthal junction with Steven's tenotomy scissors in an infero-lateral direction (Fig. 1e).

- Additional cantholysis of the lower limb of the LCT if incomplete.

- Two double-armed $6 / 0$ polyglactin 910 sutures on $1 / 2$ circle needle are pre-placed into the cut end of the LCT (firm tissue at the lateral orbital rim when grasped with toothed forceps and tugged).

- While pulling the LCT suture medially and the cut end of the lid margin laterally the tissues are overlapped and the excess marked.

- The excess lower eyelid is excised as a "V" (Fig. 1f).

- The two pre-placed sutures are then passed through the cut end of the lower lid tarsal plate, postero-anteriorly in a horizontal mattress fashion to exit on the anterior tarsal plate surface (Fig. 1g). 


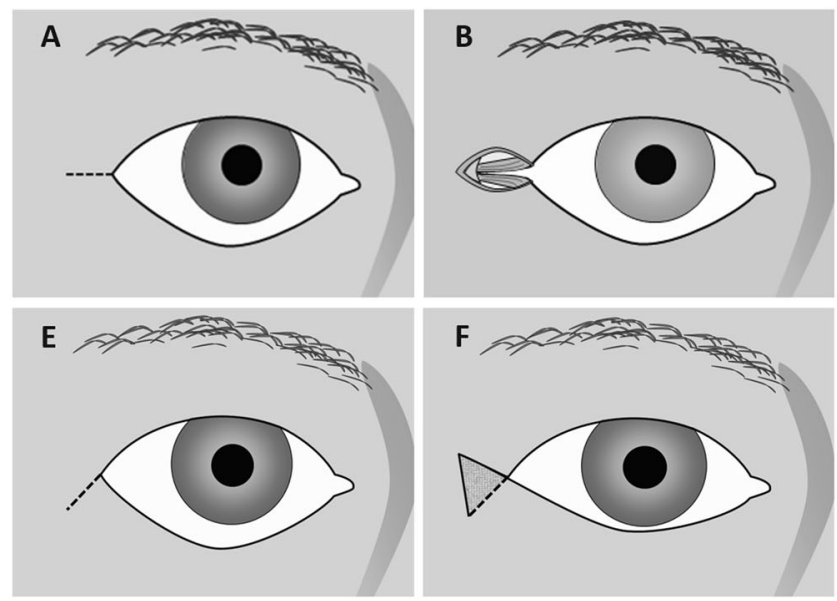

Fig. 1 Lateral tarsal strip (LTS) procedure a-d. a Lateral canthal incision, $\mathbf{b}$ the lateral canthal tendon (LCT) is cut to the orbital rim, $\mathbf{c}$ cantholysis of the lower limb of the LCT and fashioning of the LTS, d anchoring the tarsal strip to lateral orbital rim periosteum. Bick's procedure $\mathbf{e}-\mathbf{h}$. e Eyelid/lateral canthal junction incision, $\mathbf{f}$ lower eyelid

- Canthal angle reformation is achieved by the preplacement of single $7 / 0$ polyglactin 910 horizontal mattress suture. It enters the cut orbicularis to exit in the lash line and re-enter through the Meibomian orifice line of the lower lid. It is then placed similarly into the lateral canthal/upper lid (Fig. 1h).

- The previously placed $6 / 0$ tarsal sutures are then tied first and the 7/0 canthal suture second (knot buried).

- The orbicularis and skin are closed with $6 / 0$ or $7 / 0$ polyglactin 910 sutures.

\section{Statistical analysis}

All statistical analyses were performed using SPSS Statistics, version 23 software (IBM Chicago, IL, USA). Fisher's exact test was used to examine between group differences in non-continuous variables (given unequal sample size). Mann-Whitney $U$ test was used for continuous variables, which were expressed as the mean \pm SD. Statistical comparisons of patient age, gender, type of eyelid malposition, follow-up period, and grade of surgeon. All statistical tests were two sided and a $P$ value of $<0.05$ was considered statistically significant in all the tests.

\section{Results}

A total of 641 procedures (557 LTS and 84 Bick's) were performed on 504 patients (137 bilateral) by 7 consultants and their trainees. The study cohort included 286 males and 218 females. The mean age was $76.2 \pm 10.8$ years (median 78). The mean follow-up was 13.07 months (median 7 ,
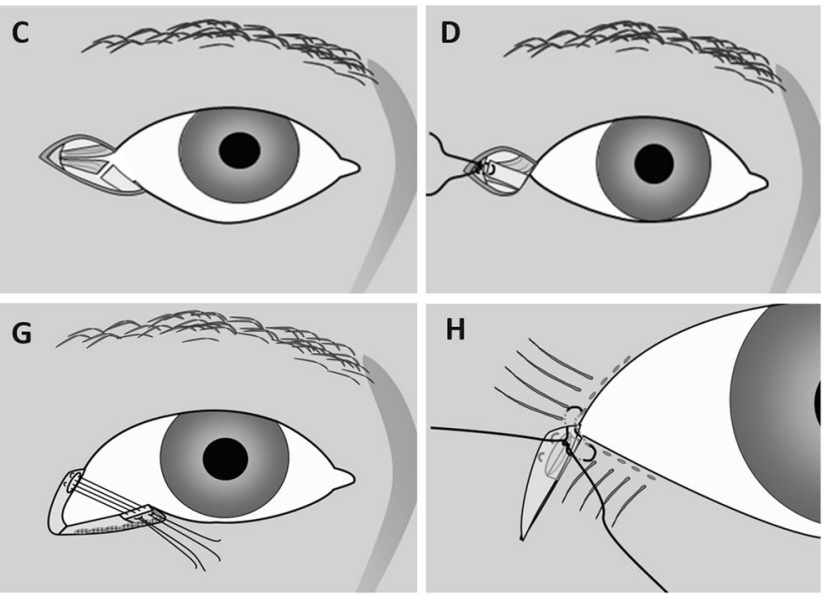

is pulled laterally and the excessive tissue is excised as a "V", g two pre-placed sutures into the cut end of the LCT are passed through the cut end of the lower lid tarsus, postero-anteriorly in a horizontal mattress fashion, $\mathbf{h}$ canthal angle reformation with horizontal mattress sutures through meibomian orifice line and lash line

Table 2 Anatomical and functional outcomes

\begin{tabular}{clll}
\hline & LTS $n(\%)$ & Bicks $n(\%)$ & $P$ value \\
\hline Anatomical outcome & & & \\
Success (overall) & $488(89.1)$ & $84(100)$ & $<0.001$ \\
$\quad$ Full correction & $446(81.4)$ & $79(94)$ & \\
Undercorrection & $34(6.2)$ & $5(6)$ & \\
Overcorrection & $8(1.5)$ & - & \\
Fail & $60(10.9)$ & 0 & $<0.001$ \\
Data missing & $9(1.4)$ & - & \\
Functional outcome & & & \\
Success (overall) & $424(82)$ & $76(95)$ & 0.002 \\
$\quad$ Resolved - complete & $311(60.2)$ & $62(77.5)$ & \\
$\quad$ Resolved - Partial & $113(21.9)$ & $14(17.5)$ & \\
Fail (overall) & $93(18)$ & $4(5)$ & 0.002 \\
$\quad$ Persistent & $92(17.8)$ & $4(5)$ & \\
$\quad$ Worse & $1(0.2)$ & - & \\
Data missing & $40(7.9)$ & $4(5)$ & \\
\hline
\end{tabular}

range $0.5-58$ months). The indications for surgery included ectropion (43.2\%), entropion (39.9\%), eyelid laxity (12.3\%), floppy eyelid syndrome (2.7\%), and others (1.9\%) (Table 1).

At last follow-up, the LTS group achieved $89.1 \%$ anatomical success (total + partial correction) compared to $100 \%$ in Bick's group $(P<0.001)$ (Table 2$)$. Functional improvement was $82 \%$ and $95 \%$ in LTS and Bick's groups, respectively $(P=0.002)$ (Table 2$)$. In terms of correcting different types of malposition, Bick's group was observed to have a higher success rate (Table 3 ). Consultants and trainee doctors achieved comparable outcomes within each group. Based on the grade of surgeon anatomical success 
Table 3 Results depending on type of malposition

\begin{tabular}{llll}
\hline Outcome & LTS $n(\%)$ & Bick $n(\%)$ & $P$ value \\
\hline Anatomical success & & & \\
Ectropion & $214(89.2)$ & $32(100)$ & 0.053 \\
Entropion & $187(90.3)$ & $47(100)$ & 0.03 \\
Lax eyelid & $67(90.5)$ & $3(100)$ & 1.0 \\
FES & $12(75)$ & $1(100)$ & 1.0 \\
Other & $8(72.7)$ & $1(100)$ & 1.0 \\
Functional success & & & \\
Ectropion & $183(81)$ & $28(96.6)$ & 0.036 \\
Entropion & $169(87.6)$ & $44(93.6)$ & 0.309 \\
Lax eyelid & $52(73.2)$ & $2(100)$ & 1.0 \\
FES & $12(75)$ & $1(100)$ & 1.0 \\
Other & $8(72.7)$ & $1(100)$ & 1.0 \\
\hline
\end{tabular}

was $89.8 \%$ for consultants vs $88.2 \%$ for trainees in the LTS group $(P=0.665)$, compared to $100 \%$ in the Bick's for both consultants and trainees. Similarly, functional outcomes did not appear to be influenced by the grade of surgeon in each study group.

Complications were relatively minor with no major long terms sequelae, nor any statistically significant difference between the two groups in terms of overall frequency of adverse events $(16.9 \%$ vs $14.2 \%, P=0.929)$. Among common complications, granuloma formation and wound dehiscence were mainly encountered mainly in the LTS group, while the infection rate was similar in both groups. The reoperation rate was $9 \%$ in the LTS group during the study period, compared to none in the Bick's group $(P=$ 0.001) (Table 4). Median interval to failure and or reoperation was 7 months.

\section{Discussion}

Horizontal eyelid laxity may be generalized or primarily affect the medial or lateral canthus. Generally, it is addressed by tightening the eyelid in its area of maximum laxity. Numerous surgical techniques have been described for correction of horizontal eyelid laxity including full thickness resection of the eyelid, lateral tarsal strip, medial, or lateral canthal tendon plication and medial canthal resection, in cases of severe medial canthal laxity [8]. There are significant advantages to performing a lid shortening procedure in the lateral canthal area, rather than in more central positions of the eyelid. These include better cosmesis, maintaining normal anatomy of the lid margin, avoidance of lid notching, or the suture-related corneal irritation and faster rehabilitation.

In 1966, Bick introduced the concept of "orbital tarsal disparity," which he recognized as an important factor for
Table 4 Complications

\begin{tabular}{llll}
\hline Complication & LTS $n(\%)$ & Bick's $n(\%)$ & $P$ value \\
\hline $\begin{array}{l}\text { Overall complication rate }(n \\
(\%)\end{array}$ & $94(16.9)$ & $12(14.2)$ & 0.929 \\
$\quad$ Granuloma & $9(1.6)$ & 0 & \\
$\quad$ Dehiscence & $22(3.9)$ & $2(2.4)$ & \\
Suture-related infection & $19(3.4)$ & $4(4.8)$ & \\
Preseptal cellulitis & $9(1.6)$ & $1(1.2)$ & \\
Orbital cellulitis & $1(0.2)$ & 0 & \\
Conjunctivitis & $13(2.3)$ & $1(1.2)$ & \\
$\quad$ Pain or irritation & $8(1.4)$ & $2(2.4)$ & \\
$\quad$ Miscellaneous & $13(2.3)$ & $2(2.4)$ & \\
Reoperation rate $(n(\%)$ & $50(9 \%)$ & 0 & 0.001 \\
\hline
\end{tabular}

development of ectropion and entropion due to a combination of eyelid laxity and age-related reduction in the volume of the orbital contents. He demonstrated that lower eyelid entropion could be temporarily corrected by injecting 2-4 ml saline into the muscle cone. Bick recommended lid shortening as a more effective modality of correcting this "orbital tarsal disparity". This simple eyelid shortening procedure involves a full thickness triangular excision of eyelid tissue at the lateral canthus. The amount of resection depends on the degree of eyelid laxity and reconstruction is achieved by reattaching the tarsal plate to the cut end of the lateral canthal tendon [4].

Tenzel et al. described the use of the lateral canthal tendon sling for correction of the involutional ectropion in 1977 [9]. This involved creation of a new lateral canthal tendon from the lateral tarsus by excising the surrounding anterior lamella and conjunctiva and attached to the lateral orbital rim periosteum through a button hole made into the upper lateral canthal tendon. Later, Anderson et al. advocated the modification of the Tenzel procedure, which they called the "tarsal strip" procedure [2,3]. Since then, this has become probably the most popular procedure for the correction of eyelid laxity.

The Bick lateral wedge resection and the lateral tarsal strip procedure are similar in so far as they are both shorten the lid laterally. However, they differ in two important respects. The first incision in the LTS from the lateral canthus to the lateral orbital rim has the potential to damage the integrity of the lateral canthal tendon (LCT). Bick's avoids the LCT altogether. Second, in the LTS tarsal plate (made up of Meibomian glands) is deliberately buried. This is not the case in Bick's procedure, which may explain why granuloma formation at the lateral canthus is not observed with this technique.

Often correction of the eyelid malposition is performed in association with adjunctive procedures. Kam et al. compared the outcomes surgery for 67 eyes with 
involutional ectropion, treated by LTS alone or LTS combined with medial spindle (MS). This study reported a functional success of $89 \%$ vs $87 \%$ and anatomical success of $78 \%$ and $82 \%$ in the LTS and LTS + MS groups, respectively [10]. Another study by Barnes et al., LTS combined with everting sutures for involutional entropion, was found to be effective in $98 \%$ of the cases [11]. Scheepers et al. conducted a randomized controlled trial comparing everting sutures (ES) with ES plus LTS for involutional entropion, which found no recurrence in the group with LTS + ES as opposed to six recurrences in the ES alone [12]. The results of these studies underline the significance of addressing horizontal eyelid laxity.

Weene reported no recurrences of involutional ectropion using Bick's procedure in 25 lids with an average follow-up of 3 years [13]. The same study also found four recurrences after 38 procedures performed for involutional entropion. Hurwitz et al. reported "successful" outcomes of correction of ectropion and entropion in over 200 cases using a modified Bick's, although no exact figures were provided [14].

A main criticism of eyelid resection at lateral canthus is the potential of causing rounding and medial displacement of the lateral canthal angle. In our study none of the patients who underwent Bick's procedure developed such deformity. This may be attributed to the preservation of an intact LCT insertion with our modified technique and the canthal reformation with the margin horizontal mattress suture. Another issue is the wound dehiscence rate, which we also found to be remarkably low in the Bick's group with only one patient developing mild post-operative wound gaping, which healed spontaneously by secondary intention and did not affect the final outcome. There were 22 cases of wound dehiscence in the LTS group. Six of these patients required re-do surgery (five necessitated re-do LTS and one had lateral canthopexy).

This study has several limitations. The main methodological drawbacks are its retrospective design with potential bias in patient selection and lack of masked evaluation of the outcomes, unequal sample sizes, and different grades of surgeons. Additionally, there were some differences in baseline characteristics in terms of the proportion of different types of eyelid malposition. A significant group of patients were discharged after a short follow-up. However, both hospital are regional referral centers and the patients are usually referred back to these units for further management of complications or recurrence. Hence, this study provides real-world outcomes in the setting of a national healthcare system. One may also attribute the higher success rate in the Bick's group to the fact that the majority of the procedures were performed by a consultant. However, the grade of surgeon did not seem to have a significant influence on the LTS group outcomes. A prospective randomized controlled trial comparing the outcomes of the two procedures may be able to clarify some of these issues.

In summary, this study reports the largest cohort of eyelid malposition surgery comparing the outcomes of two different techniques for lid margin shortening. Although Bick's procedure achieved significantly better anatomical and functional outcomes compared to LTS, the samples were unequal and not randomized. We conclude that lateral Bick's shortening is a good alternative to LTS in addressing horizontal eyelid laxity. It is simpler to perform and avoids the additional risk of granuloma formation (presumably caused by burying meibomian gland tissue) in the LTS procedure.

\section{Summary}

\section{What was known before}

- Horizontal laxity is an important factor in eyelid malposition.

- Lateral tarsal strip is a popular and successful procedure for correction of horizontal eyelid laxity.

- Bick's procedure offers the advantages of the LTS, as well as being a technically simpler operation.

\section{What this study adds}

- In this study, Bick's procedure achieved significantly better anatomical and functional outcomes compared to lateral tarsal strip.

- Bick's procedure minimizes the risk of granuloma formation (presumably caused by burying meibomian gland tissue) in the LTS procedure.

Author contributions All authors listed have contributed sufficiently to the project to be included as authors, and all those who are qualified to be authors are listed in the author byline. All authors agree to be accountable for all aspects of the work in ensuring that questions related to the accuracy or integrity of any part of the work are appropriately investigated and resolved. The manuscript has been read and approved by all the authors, and the requirements for authorship as stated above have been met.

\section{Compliance with ethical standards}

Conflict of interest The authors declare that they have no conflict of interest.

\section{References}

1. Collin JRO. A manual of systematic eyelid surgery. 2nd ed. New York: Churchill Livingstone; 1989.

2. Anderson RL, Gordy DD. The tarsal strip procedure. Arch Ophthalmol. 1979;97:2192-6. 
3. Jordan DR, Anderson RL. The lateral tarsal strip revisited. The enhanced tarsal strip. Arch Ophthalmol. 1989;107:604-6.

4. Bick MW. Surgical management of orbital tarsal disparity. Arch Ophthalmol. 1966;75:386-9.

5. Nowinski TS, Anderson RL. The medial spindle procedure for involutional medial ectropion. Arch Ophthalmol. 1985;103: 1750-3.

6. Collin JRO. A manual of systematic eyelid surgery. 3nd ed. New York: Churchill Livingstone; 2006. p. 37.

7. Jones LT, Reeh MJ, Wobig JL. Senile entropion. A new concept for correction. Am J Ophthalmol. 1972;74:327-9.

8. Collin JRO. A manual of systematic eyelid surgery. 3nd ed. New York: Churchill Livingstone; 2006. p. 59-60.

9. Tenzel RR, Buffam FV, Miller GR. The use of the "lateral canthal sling" in ectropion repair. Can J Ophthalmol. 1977;12: 199-202.
10. Kam KY, Cole CJ, Bunce C, Watson MP, Kamal D, Olver JM. The lateral tarsal strip in ectropion surgery: is it effective when performed in isolation? Eye. 2012;26:827-32.

11. Barnes JA, Bunce C, Olver JM. Simple effective surgery for involutional entropion suitable for the general ophthalmologist. Ophthalmology. 2006;113:92-6.

12. Scheepers MA, Singh R, Ng J, Zuercher D, Gibson A, Bunce C, Fong K, Michaelides M, Olver J. A randomized controlled trial comparing everting sutures with everting sutures and a lateral tarsal strip for involutional entropion. Ophthalmology. 2010;117:352-5.

13. Weene LE. Bick procedure for correction of senile entropion and ectropion. Ophthalmic Surg. 1977;8:40-1.

14. Hurwitz JJ, Mishkin SK, Rodgers KJ. Modification of Bick's procedure for treatment of eyelid laxity. Can J Ophthalmol. 1987;22:262-5. 